\title{
SCIENTIFIC REPORTS

\section{Using the maximum clustering heterogeneous set-proportion to select the maximum window size for the spatial scan statistic}

\author{
Wei Wang ${ }^{1,4}$, Tao Zhang ${ }^{1,4}$, FeiYin ${ }^{1}$, Xiong Xiao ${ }^{1}$, Shiqi Chen ${ }^{2}$, Xingyu Zhang ${ }^{3}$, Xiaosong Li ${ }^{1}$ \& \\ Yue $\mathrm{Ma} \mathbb{1}^{1 *}$
}

The spatial scan statistic has been widely used to detect spatial clusters that are of common interest in many health-related problems. However, in most situations, different scan parameters, especially the maximum window size (MWS), result in obtaining different detected clusters. Although performance measures can select an optimal scan parameter, most of them depend on historical prior or true cluster information, which is usually unavailable in practical datasets. Currently, the Gini coefficient and the maximum clustering set-proportion statistic (MCS-P) are used to select appropriate parameters without any prior information. However, the Gini coefficient may be unstable and select inappropriate parameters, especially in complex practical datasets, while the MCS-P may have unsatisfactory performance in spatial datasets with heterogeneous clusters. Based on the MCS-P, we proposed a new indicator, the maximum clustering heterogeneous set-proportion (MCHS-P). A simulation study of selecting the optimal MWS confirmed that in spatial datasets with heterogeneous clusters, the MWSs selected using the MCHS-P have much better performance than those selected using the MCS-P; moreover, higher heterogeneity led to a larger advantage of the MCHS-P, with up to $538 \%$ and $69.5 \%$ improvement in the Youden's index and misclassification in specific scenarios, respectively. Meanwhile, the MCHS-P maintains similar performance to that of the MCS-P in spatial datasets with homogeneous clusters. Furthermore, the MCHS-P has significant improvements over the Gini coefficient and the default 50\% MWS, especially in datasets with clusters that are not far from each other. Two practical studies showed similar results to those obtained in the simulation study. In the case where there is no prior information about the true clusters or the heterogeneity between the clusters, the MCHS-P is recommended to select the MWS in order to accurately identify spatial clusters.

With the development of geographic information systems, the global positioning system and remote sensing, a large number of health-related datasets with geographic locations are being collected. Accurately identifying the spatial variability, such as the difference in disease incidence between different locations, plays an important role in the elucidation of the potential causes of illnesses, allocation of limited health resources, formulation of rational public health policies and exploration of the characteristics of health-related problems. Kulldorff's spatial scan statistic (SSS) ${ }^{1}$ is one of the most commonly used methods ${ }^{2-5}$ for identifying such a difference, namely, the region that is significantly different from the other regions and is known as a cluster. However, even when employing the SSS for the same dataset, different scan parameters will usually result in different results with different accuracy. For instance, in Ribeiro's simulation study ${ }^{6}$, different parameters led to highly different powers, sensitivity and positive predictive values in the same data, with the largest differences reaching $0.9404,0.9369$, and 0.825 , respectively. Tango's simulation study ${ }^{7}$ showed that the difference in the power between different parameters reached 0.596 ( 0.738 vs 0.142$)$. In these scan parameters, the maximum spatial window size (MWS), commonly defined as a percentage of the maximum population in a single cluster, has very strong effect on the detected result ${ }^{6,8}$. The

${ }^{1}$ West China School of Public Health and West China Fourth hospital, Sichuan University, Chengdu, China. ${ }^{2}$ Women and Children's Health Management Department, Sichuan Provincial Hospital for Women and Children, Chengdu, China. ${ }^{3}$ Department of Systems, Populations and Leadership, University of Michigan, School of Nursing, Ann Arbor, United States. ${ }^{4}$ These authors contributed equally: Wei Wang and Tao Zhang. *email: gordonrozen@qq.com 
MWS is set to the default value of $50 \%$ in most studies and in some cases this leads to detecting an overly large region falsely covering regions between several small real clusters. Smaller MWSs are also selected for practical reasons, including limited resources for intervention ${ }^{6}$, special terrain ${ }^{9}$, spatial location discontinuity ${ }^{10}$, and other reasons ${ }^{11-13}$. However, different MWSs lead to different sizes, locations and numbers of the detected clusters in the same data ${ }^{8,14-16}$. Therefore, selecting an appropriate MWS for the SSS is important for accurate cluster identification.

The indicators used to select the MWS mainly include the classic performance measures such as sensitivity, specificity, positive predictive value (PPV), Youden's index (YDI), misclassification and the recently developed maximum clustering set-proportion (MCS-P) statistic ${ }^{17}$. However, due to the lack of historical prior or true cluster information in practice, these classic performance measures usually cannot be calculated. The MCS-P depends only on the applied spatial data and provides a solution in practice without using any true cluster information or historical prior data. Although for datasets with homogeneous clusters, i.e., clusters having similar relative risks (RR), selecting the MWS with the MCS-P shows good performance compared to that obtained by using the default MWS values, the MCS-P does not perform well for datasets that include significantly heterogeneous clusters ${ }^{17}$. Furthermore, many researchers also use the Gini coefficient to select the maximum reported spatial window size (MRWS) to avoid reporting an overly large cluster and increase the accuracy of the detected clusters $^{18,19}$. The MRWS is the maximum reported population in clusters detected based on a pre-selected fixed MWS larger than the MRWS. Although the Gini coefficient is not used to select the MWS, it can achieve results similar to those obtained by selecting the MWS and it therefore has become a commonly used indicator due to its implementation in the SaTScan software. However, in our exploratory studies, we found that the Gini coefficient may be unstable, is still likely to detect clusters that are much larger than the real clusters, and will even obtain the same results as the 50\% MWS, especially for datasets with clusters that are not far from each other, as will be described in detail in the methodology section. Therefore, considering the general existence of complex spatial distribution patterns in reality, especially in health-related datasets, a more efficient indicator is necessary for selecting the MWS.

This study proposed a new indicator based on MCS-P, called the maximum clustering heterogeneous set-proportion (MCHS-P), for selecting an appropriate MWS for the SSS. The MCHS-P can identify potential heterogeneous clusters accurately and depends only on the detected clusters and the applied dataset without any historical prior or true cluster information. Section 2 gives the definition of the MCHS-P and a brief discussion about the limitations and uncertainty for the use of the Gini coefficient that is an alternative indicator for use in practical datasets. Section 3 presents a simulation study and a practical study for the comparison of the MCHS-P with the MCS-P in the selection of MWS for the SSS in different datasets with either homogeneous or heterogeneous clusters. The results obtained using the Gini coefficient and the default $50 \% \mathrm{MWS}$ are also provided as reference data. Sections 4 and 5 provide a discussion of these results and the conclusion of the paper, respectively.

\section{Methods}

The SSS and the MCS-P. The SSS is used to identify the likely clusters in spatial datasets. First, it searches for a set of windows under a specified scan parameter. Then, for each window (z), under the alternative hypothesis ( $\mathrm{z}$ is a cluster) and the null hypothesis (no cluster exists), the logarithm of the likelihood ratio $L L R(\mathrm{z})$ is constructed under the Poisson distribution as follows:

$$
L L R(\mathrm{z})=\ln \left\{\left(\frac{c_{z}}{n_{z}}\right)^{c_{z}}\left(\frac{C-c_{z}}{C-n_{z}}\right)^{C-c_{z}}\right\},
$$

where $C$ is the total number of the observed cases in the entire study area. $n_{z}$ and $c_{z}$ are the expected cases under the null hypothesis and the observed cases in $\mathrm{z}$, respectively. Finally, the windows with an $L L R(\mathrm{z})$ greater than the critical value obtained from Monte Carlo simulations are selected as the detected significant clusters. Usually, clusters with RR either larger or smaller than 1 are considered for different purposes.

Different MWSs lead to different sets of windows ( $\mathrm{z}$ ) and then different detected clusters. The MCS-P is designed to select the optimal result from the results obtained with different MWSs. First, the MCS-P merges all the detected clusters under a certain single parameter into a union cluster to construct the union $L L R(\mathrm{z})$ that reflects the capability of the dataset to support the current result. To get a more stable average value in simulation datasets, the approximately maximal $L L R$ based on the most clustering set (MCS) is used to adjust the union $L L R$ to the MCS-P. The MCS is composed of all the units with RR either larger or smaller than 1 for the corresponding purpose. Thus, its corresponding $L L R$ will be constant in a specified dataset and not necessary in practical application. Such an $L L R$ represents the approximately maximal value that the $L L R$ can reach when the geographic positions of all the spatial units in the study region are ignored.

$$
\begin{gathered}
z_{i 0}=\cup_{j} z_{i j}, \\
\operatorname{LLR}\left(z_{i 0}\right)=\ln \left\{\left(\frac{c_{z_{i 0}}}{n_{z_{i 0}}}\right)^{c_{z_{i 0}}}\left(\frac{C-C_{z_{i 0}}}{C-n_{z_{i 0}}}\right)^{C-c_{z_{i 0}}}\right\}, \\
z_{M C S}=\cup\left\{x: x \in G, p_{x}>q_{x}\right\} \text { or } z_{M C S}=\cup\left\{x: x \in G, p_{x}<q_{x}\right\},
\end{gathered}
$$




$$
\operatorname{MCS}-\mathrm{P}=\frac{\operatorname{LLR}\left(z_{i 0}\right)}{\operatorname{LLR}\left(z_{M C S}\right)},
$$

where $z_{i j}$ is the $j$ th detected cluster under the $i$ th MWS. $z_{i 0}$ is the union cluster, $x$ is a spatial unit in the entire study area $G$, and $p_{x}$ and $q_{x}$ are the probabilities of an event occurrence in $x$ and out of $x$, respectively. $z_{M C S}$ is a window composed of all the units in the MCS. A larger MCS-P means that the corresponding detected clustering set is more supported by the applied dataset.

As observed from Eq. (1), the MCS-P assumes that all the detected clusters under a selected MWS have the same RR. However, in practical datasets such as chronic disease datasets, different clusters may have different RR due to the different environmental and socioeconomic factors. Therefore, the MCS-P may lead to unsatisfactory performance in such datasets, as has been reported in previous studies ${ }^{17}$.

An improved statistic based on the MCS-P. To improve the performance of the MCS-P in spatial datasets with heterogeneous clusters, the union of detected clusters is reconstructed to fit the potential heterogeneity between the clusters. A very easy approach is to assume that each cluster can have a different RR. However, with a small maximal window size, a single cluster may be detected as a set of small, spatially continuous clusters. Assuming such homogeneous clusters to have different RR may lead to overfitting. Therefore, a constraint is introduced to limit such overfitting. Based on the common knowledge that nonadjacent clusters are usually independent for spatial isolation ${ }^{20}$, the detected clusters under a single certain selected parameter are reconstructed into a set of regions according to their spatial contiguity. Given that the number of detected clusters under the $i$ th selected parameter is $y$; then, with only contiguous clusters merged into one clustering region, $y$ detected clusters are merged into $k(k \leq y)$ merged clusters. The $k$ merged clusters comprise the detected potential heterogeneous clustering set $S_{i}(k)$ under the $i$ th selected parameter. The corresponding union $\operatorname{LLR} L L R\left(S_{i}(k)\right)$ is calculated as:

$$
\begin{aligned}
& S_{i}(k)=\left\{z_{i m 1}, z_{i m 2}, \ldots, z_{i m k}\right\}, \\
& \operatorname{LLR}\left(S_{i}(k)\right)=\ln \left\{\left(\frac{c_{z_{i m 1}}}{n_{z_{i m 1}}}\right)^{c_{z_{i m 1}}}\left(\frac{c_{z_{i m 2}}}{n_{z_{i m 2}}}\right)^{c_{z_{i m 2}}} \ldots\left(\frac{c_{z_{i m k}}}{n_{z_{i m k}}}\right)^{c_{z_{i m k}}}\left(\frac{C-\sum_{j=1}^{k} c_{Z_{i m j}}}{C-\sum_{j=1}^{k} n_{z_{i m j}}}\right)^{C-\sum_{j=1}^{k} c_{z_{i m j}}}\right\} .
\end{aligned}
$$

where $z_{i m j}$ represents the $j$ th merged cluster under the $i$ th parameter. Such an $L L R\left(S_{i}(k)\right)$ takes into consideration both the potential heterogeneity among the detected nonadjacent clusters and the similarity among the spatial contiguous units, and it also limits the overfitting.

To get a more stable average value in simulation datasets, an approximately maximal $L L R$ based on the most clustering heterogeneous set (MCHS) is adopted. Unlike for the MCS, the spatial units in the MCHS are permitted to have different probabilities of event occurrence if they are not adjacent. Specifically, the MCS is separated into several subsets based on whether the spatial units are contiguous. The units in the same subset share the same estimated probability of event occurrence. Thus, the units in different subsets may have different probabilities. These heterogeneous subsets compose the MCHS. The corresponding union LLR of the MCHS is LLR(MCHS).

$$
\begin{aligned}
& \text { MCHS }=\left\{z_{\text {subset } 1}, z_{\text {subset } 2}, \ldots, z_{\text {subsetw }}\right\} \\
& L L R(\mathrm{MCHS})=\ln \left\{\left(\prod_{j=1}^{w}\left(\frac{c_{z_{\text {subsetj }}}}{n_{z_{\text {subsetj }}}}\right)^{c_{z_{\text {subset }}}}\right)\left(\frac{C-\sum_{j=1}^{w} c_{Z_{\text {subsetj }}}}{C-\sum_{j=1}^{w} n_{z_{\text {subsetj }}}}\right)^{\left.C-\sum_{j=1}^{w} c_{Z_{\text {subsetj }}}\right\}},\right.
\end{aligned}
$$

where $w$ is the number of subsets in the MCHS and $z_{\text {subsetj }}$ represents the window composed of the spatial units in the jth subset. The denominator $L L R(\mathrm{MCHS}$ ) represents the approximately maximal union $L L R$ in the specified $G$ with potential heterogeneous nonadjacent clusters, which is a constant in a specified dataset and not necessary in practical application. Then, the improved indicator MCHS-P is given as:

$$
\text { MCHS-P }=\frac{L L R\left(S_{i}(k)\right)}{L L R(\mathrm{MCHS})} .
$$

The MCHS-P reflects the ratio of the union LLR between the detected potential heterogeneous clustering set and the most clustering heterogeneous set in $G$. With the detected clusters reconstructed into a potential heterogeneous clustering set, the MCHS-P adapts to spatial datasets with homogeneous clusters and heterogeneous clusters and is still an approximate relative indicator that depends only on the detected clusters and the applied dataset rather than on any historical prior or true cluster information.

The Gini coefficient for MRWS selection. To select the MRWS, the Gini coefficient measures the difference of the case distribution in the clusters and non-clusters based on the cumulative cases and expected cases in the clusters. First, the clusters detected under a certain MRWS are listed in descending order by RR. Second, the cumulative percentage of cases and the cumulative percentage of expected cases for each cluster point are computed. For example, as shown in Fig. 1(a), points $\mathrm{O}$ and $\mathrm{C}$ are $(0,0)$ and $(100,100)$, respectively, so that the reciprocal of the slope of $\mathrm{OC}$ measures the expected $R R$, i.e., $R R=1$. Two clusters $\left(A_{1}\right.$ and $\left.A_{2}\right)$ are detected and the 


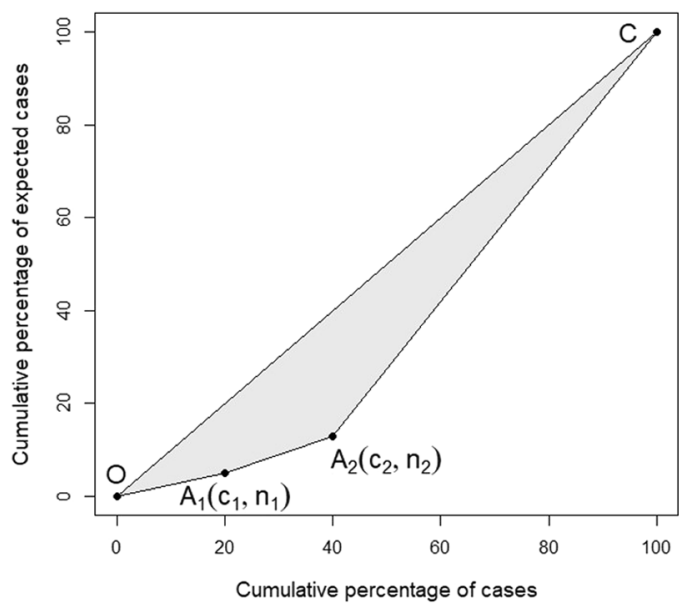

(a)

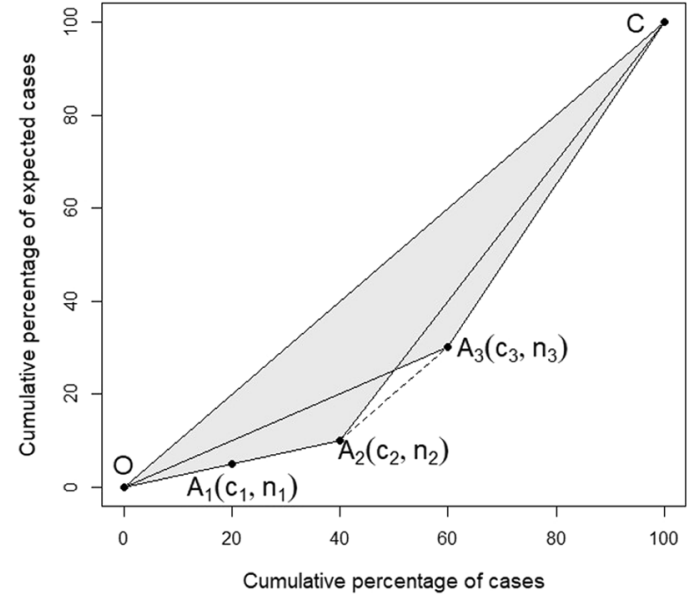

(b)

Figure 1. Illustration of the Gini coefficients. Subfigure (a) is for a cluster model with two clusters (A1 and A2). Subfigure (b) is a comparison between the two Gini coefficients of the two detected results. The first coefficient is for two homogeneous small clusters (A1 and A2), and the second is for a large cluster (A3).

reciprocal of the slope of $O A_{1}$ is larger than $A_{1} A_{2}$, meaning that the RR of $A_{1}$ is larger than $A_{2} \cdot c_{1}$ and $n_{1}$ are the observed and expected cases in cluster $A_{1}$, respectively; $c_{2}$ is the sum of the observed cases in $A_{1}$ and $A_{2}$ and $n_{2}$ is the sum of the expected cases in $A_{1}$ and $A_{2}$. The Gini coefficient is equal to two times the area of $\mathrm{OA}_{1} \mathrm{~A}_{2} \mathrm{C}$, namely, Gini $\left(\mathrm{A}_{1} \mathrm{~A}_{2}\right)=2 \times S_{\mathrm{OA}_{1} \mathrm{~A}_{2} \mathrm{C}}$. Finally, the MRWS with the largest Gini coefficient is selected as the optimal MRWS, as shown in detail by Han et al. ${ }^{18}$.

Although the Gini coefficient provides a better detected result for the SSS than the default MWS in many situations, in our exploratory studies, we found that the improvement may be unstable and insignificant, especially for datasets with homogeneous clusters that are not far from each other. A typical case is shown in Fig. 1(b), where the Gini coefficients for the two detected results are presented. The first detected result includes two real homogeneous small clusters $\left(A_{1}\right.$ and $\left.A_{2}\right)$ and the other includes a large detected cluster $A_{3}$ that is a superset of $A_{1}$ and $A_{2}$. When the incidence of these spatial units in $A_{3}$ but out of $A_{1}$ and $A_{2}$ is equal to the expected RR, line $A_{2} A_{3}$ is parallel to $\mathrm{OC}$ and the area of $\mathrm{OA}_{2} \mathrm{~A}_{3}$ is equal to that of $\mathrm{CA}_{2} \mathrm{~A}_{3}$, namely, $\operatorname{Gini}\left(\mathrm{A}_{1} \mathrm{~A}_{2}\right)=\operatorname{Gini}\left(\mathrm{A}_{3}\right)$. Thus, a small random fluctuation of $R R$ in the spatial units in $A_{3}$ but out of $A_{1}$ and $A_{2}$ will lead to a smaller slope of $A_{2} A_{3}$ than that of OC. As a result, Gini $\left(\mathrm{A}_{1} \mathrm{~A}_{2}\right)$ will be smaller than $\operatorname{Gini}\left(\mathrm{A}_{3}\right)$ and $\mathrm{A}_{3}$ will be falsely detected as clusters.

Therefore, selection of the MRWS using the Gini coefficient may not obtain good results in the complex practical datasets. Nevertheless, this approach is still commonly used owing to its convenient implementation in the SaTScan software.

\section{Result}

Simulation study. To validate the capability of the MCHS-P to select an appropriate MWS for the SSS in datasets with heterogeneous clusters, we employed a series of simulation datasets with different heterogeneities among the clusters to compare the performance of the MCHS-P with that of the MCS-P. Additionally, to evaluate the risk of the MCHS-P selecting worse parameters than the MCS-P because of overfitting in datasets with homogeneous clusters, we also used a series of simulation scenarios with different random fluctuations out of the homogeneous cluster, in which a higher random fluctuation is more likely to lead to a false detected cluster. The results with the default $50 \%$ MWS and Gini coefficient were also provided as reference data.

Simulation data. Simulation datasets are taken from Kulldorff's benchmark data ${ }^{21}$ that have been commonly used for the evaluation of SSS with different scan parameters ${ }^{22-24}$ and the validation of the MCS-P in selecting $\mathrm{MWS}^{17}$. These simulation datasets are based on a real dataset for breast cancer mortality during 1988-1992 that consists of the data for 29,535,210 women in 245 counties in the northeastern USA.

Two total case numbers, 600 and 6000, were considered. For each number, three different sets of concentric circle clusters were constructed that included women located in three different areas, such as rural, mixed, and urban areas. Each of these three sets contains clusters with different sizes, for example, 1, 2, 4, 8, or 16 counties. Based on these clusters, a total of 50 simulation scenarios were built. The details are shown in Table 1. For each scenario, 10,000 datasets were generated. All the datasets are available at the SaTScan website ${ }^{25}$.

These 50 scenarios include 10 simulation scenarios with two heterogeneous equal-sized clusters located in the rural and urban areas, respectively; 10 scenarios with three heterogeneous equal-sized clusters located in the rural, mixed and urban areas, respectively; and 30 scenarios with a single cluster that can be deemed as scenarios with homogeneous clusters, since there is no heterogeneity for a single cluster. For the heterogeneous cluster models, the scenarios with a smaller cluster size, two clusters and 600 cases are more heterogeneous than those with a larger cluster size, three clusters and 6,000 cases, respectively. 


\begin{tabular}{|c|c|c|c|c|c|c|c|c|c|c|c|c|c|c|}
\hline \multirow{2}{*}{\multicolumn{2}{|c|}{ Location }} & \multicolumn{9}{|c|}{ Homogeneous clusters } & \multicolumn{4}{|c|}{ Heterogeneous clusters } \\
\hline & & \multicolumn{3}{|l|}{ Rural } & \multicolumn{3}{|l|}{ Mixed } & \multicolumn{3}{|l|}{ Urban } & \multicolumn{2}{|c|}{ Two clusters } & \multicolumn{2}{|c|}{ Three clusters } \\
\hline Cases & Size & POP & RR & $\mathbf{P}$ & POP & RR & $\mathbf{P}$ & POP & RR & $\mathbf{P}$ & POP & $\mathbf{H}$ & POP & $\mathbf{H}$ \\
\hline \multirow{5}{*}{600} & 1 & 2675 & 192.89 & 1.998 & 710196 & 2.85 & 1.946 & 786178 & 2.73 & 1.941 & 788853 & 190.16 & 1499049 & 190.16 \\
\hline & 2 & 22911 & 27.03 & 1.992 & 817050 & 2.70 & 1.943 & 1072181 & 2.43 & 1.932 & 1095092 & 24.6 & 1912142 & 24.6 \\
\hline & 4 & 132343 & 7.05 & 1.979 & 1108440 & 2.40 & 1.931 & 2953077 & 1.81 & 1.881 & 3085420 & 5.24 & 4193860 & 5.24 \\
\hline & 8 & 204829 & 5.35 & 1.971 & 1352284 & 2.24 & 1.923 & 5018909 & 1.63 & 1.836 & 5223738 & 3.72 & 6576022 & 3.72 \\
\hline & 16 & 360275 & 3.9 & 1.961 & 1684327 & 2.1 & 1.914 & 7627173 & 1.53 & 1.785 & 7987448 & 2.37 & 9671775 & 2.37 \\
\hline \multirow{5}{*}{6000} & 1 & 2675 & 23.73 & 20.27 & 710196 & 1.45 & 20.09 & 786178 & 1.43 & 20.08 & 788853 & 22.3 & \begin{tabular}{|l|}
1499049 \\
\end{tabular} & 22.3 \\
\hline & 2 & 22911 & 4.96 & 20.25 & 817050 & 1.42 & 20.09 & 1072181 & 1.36 & 20.05 & 1095092 & 3.6 & 1912142 & 3.6 \\
\hline & 4 & 132343 & 2.21 & 20.21 & 1108440 & 1.36 & 20.04 & 2953077 & 1.22 & 19.88 & 3085420 & 0.99 & 4193860 & 0.99 \\
\hline & 8 & 204829 & 1.92 & 20.18 & 1352284 & 1.32 & 20.02 & 5018909 & 1.17 & 19.73 & 5223738 & 0.75 & 6576022 & 0.75 \\
\hline & 16 & 360275 & 1.66 & 20.15 & 1684327 & 1.29 & 19.99 & 7627173 & 1.15 & 19.57 & 7987448 & 0.51 & 9671775 & 0.51 \\
\hline
\end{tabular}

Table 1. Simulation scenarios with homogeneous/heterogeneous clusters. Note: POP is the total population in the clusters. RR is the relative risk of the clusters. $\mathrm{P}$ is the incidence $\left(\times 10^{-5}\right)$ out of the clusters. Because the case numbers follow a Poisson distribution under the null hypothesis, the incidence rate also reflects random fluctuation out of the clusters. $\mathrm{H}$ is the difference between the maximal $\mathrm{RR}$ and minimal $\mathrm{RR}$ that reflects the strength of the heterogeneity among the clusters. Heterogeneity among the clusters becomes lower as the cluster number, total case number and cluster size grow.

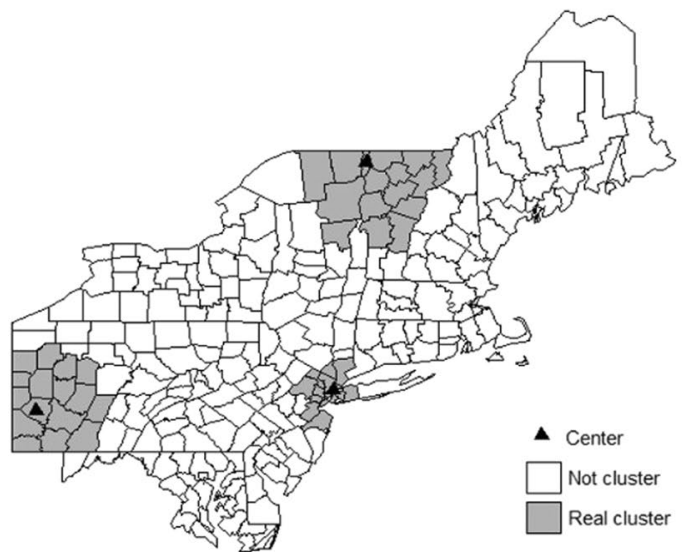

(a)

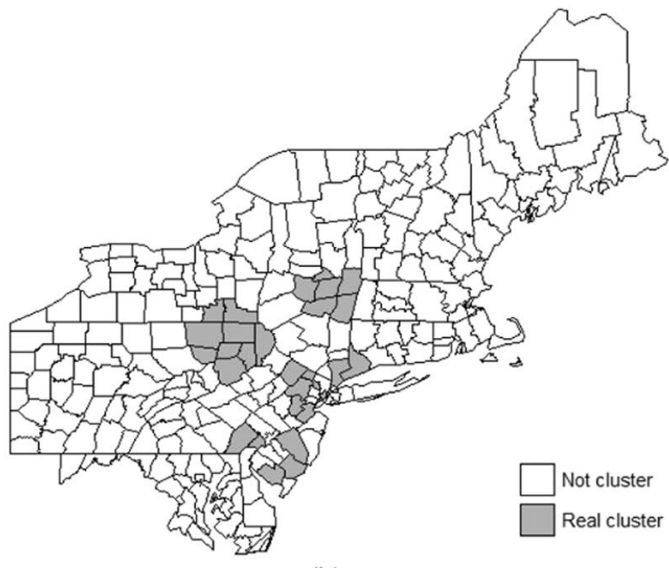

(b)

Figure 2. Positions of real clusters. Subfigure (a) is the position of real clusters with 16 counties for each in Kulldorff's benchmark datasets and the solid triangles are the centres for the three types of clusters such as those located in rural, mixed and urban areas. Subfigure (b) shows the positions of the real clusters in the additional scenario in which the clusters are not far away from each other.

These scenarios are referred to as "total case numbers-cluster location-cluster size" for short. For example, 6000-rural-1 refers to the scenario with a total case number of 6,000, one cluster located in the rural area, and one county for the cluster; 6000-two-4 refers to the scenario with a total case number of 6,000, two clusters located in the rural and urban areas, and four counties for each cluster.

In addition, as shown in Fig. 2(a), the three clusters with different centres in the above-described scenarios are so far away from each other, for example with the away region containing more than $50 \%$ of the total population at risk, that even the 50\% MWS could not cover any two clusters and therefore, the false positive rate will be underestimated in such datasets. Considering that the clusters that are not located so far apart are common in practical datasets, we built an additional scenario that is similar to the complex real datasets, called the complex scenario available from Supplementary File 1, in which the artificial clusters are not located far apart, as shown in Fig. 2(b). In the complex scenario, a total of 6,000 cases were included and the RR in these clusters were set to 1.55 .

Performance comparison. From 50 different MWSs in the range of $1-50 \%$ with an interval of $1 \%$ population at risk, the MCHS-P and the MCS-P were used to select their own optimal MWS under the most commonly used circle window. Only significant clusters with a P-value less than 0.05 and no geographic overlap with more likely clusters were considered; these parameters were also set for the detected result obtained from the Gini coefficient and the 50\% MWS. The SaTScan9.4 free software was selected as the clustering detection tool.

Subsequently, MWSs with the largest the MCHS-P and the MCS-P were selected as their own optimal parameters, respectively. The SSS's performance characteristics obtained using the MCHS-P, MCS-P, 50\% MWS, and 


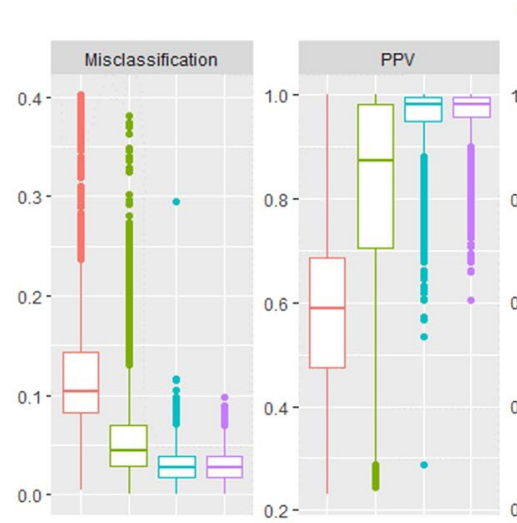

\section{The complex scenario}
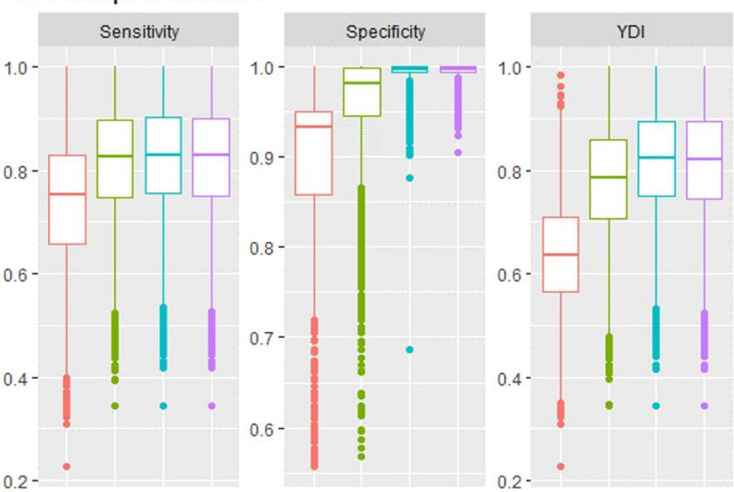

Method

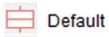

$\biguplus$ Gini

MCHS-P

MCS-P

The boxplot of the classic performance measures from the MCHS-P, MCS-P, 50\% MWS and Gini coefficient

in the complex scenario.

Figure 3. Comparison of the results between the MCHS-P, MCS-P, 50\% MWS and Gini coefficient in the complex scenario. It is observed that the MCS-P and the MCS-P have more stable and better performance than the $50 \%$ MWS and the Gini coefficient.

Gini coefficient were compared using the average values of 5 commonly used classic performance measures over replicas: sensitivity, specificity, positive predictive value (PPV), Youden's index (YDI) and misclassification ${ }^{22,26-29}$. Additionally, we calculated the average values of the MCHS-P, the MCS-P and each classic performance measure for each MWS over replicas to evaluate the relationships of the MCHS-P and the MCS-P with the classic performance measures.

Of these five classic performance measures, sensitivity reflects the capacity to correctly identify true clusters. Specificity and PPV reflect the capability to correctly identify the units out of clusters. YDI and misclassification reflect both aspects. Because the measures based on the population may be more robust ${ }^{22}$, the five classic performance measures were calculated as follows:

$$
\begin{aligned}
& \text { sensitivity }=\frac{a}{a+b}, \\
& \text { specificity }=\frac{d}{d+c}, \\
& \mathrm{PPV}=\frac{a}{a+c}, \\
& \mathrm{YDI}=\text { sensitivity }+ \text { specificity }-1, \\
& \text { misclassification }=\frac{b+c}{a+b+c+d},
\end{aligned}
$$

where $\mathrm{a}, \mathrm{b}, \mathrm{c}$ and $\mathrm{d}$ represent the total population in the four types of spatial units (units in true clusters and detected clusters, units in true clusters and not in detected clusters, units in detected clusters and not in true clusters and units in neither detected clusters nor true clusters, respectively).

Comparison between the four methods. The average sensitivity, specificity, PPV, YDI and misclassification over replicas from the MCHS-P, MCS-P, 50\% MWS, and Gini coefficient were compared, and the detail is seen in Supplementary File 2. Generally, in 50 types of benchmark datasets, the 50\% MWS and the Gini coefficient performs well but slightly poorer than the MCHS-P, especially in the scenarios with multiple clusters or clusters covering large population. However, in the complex scenario, as shown in Figs. 3 and 4, the default 50\% MWS performs poorly due to its high false positive rate. The Gini coefficient makes a mild and unstable improvement, while the MCHS-P still has a consistently good performance. The MCS-P exhibits the same performance as found in the previous study, that is to say its performance is good in scenarios with homogeneous clusters and is poor in scenarios with heterogeneous clusters, especially for the highly heterogeneous clusters such as 600-two-1, 600-two-2, 600-three-1, 6000-two-1, and 6000-three-1. As expected, the MCHS-P has much improvements in the heterogeneous scenarios and maintains similar performance with the MCS-P in the homogeneous scenarios.

Specifically, in the 20 scenarios with heterogeneous clusters (multiple clusters), as the heterogeneity increases, corresponding to the decrease in the cluster size, the total case number and cluster number, the MCHS-P become more advantageous compared to the MCS-P, and the largest improvements in sensitivity, YDI and misclassification reach $540 \%, 538 \%$ and $69.5 \%$ ( 0.9047 vs $0.1413,0.9000$ vs 0.1410 and 0.0071 vs 0.0232 ), respectively, in 600-two-1, whereas the specificity and PPV values decline slightly by $0.44 \%$ and $4.58 \%$ ( 0.9953 vs 0.9997 and 0.9442 vs 0.9895 ), respectively. Although the $50 \%$ MWS and the Gini coefficient also show a good performance for the large separation between clusters, they are still outperformed by the MCHS-P. An examination of the data presented in Table 2 shows that the MCHS-P achieves optimal aggregative indicators such as YDI and misclassification in all 20 scenarios. In the 30 scenarios with homogeneous clusters (a single cluster), the 

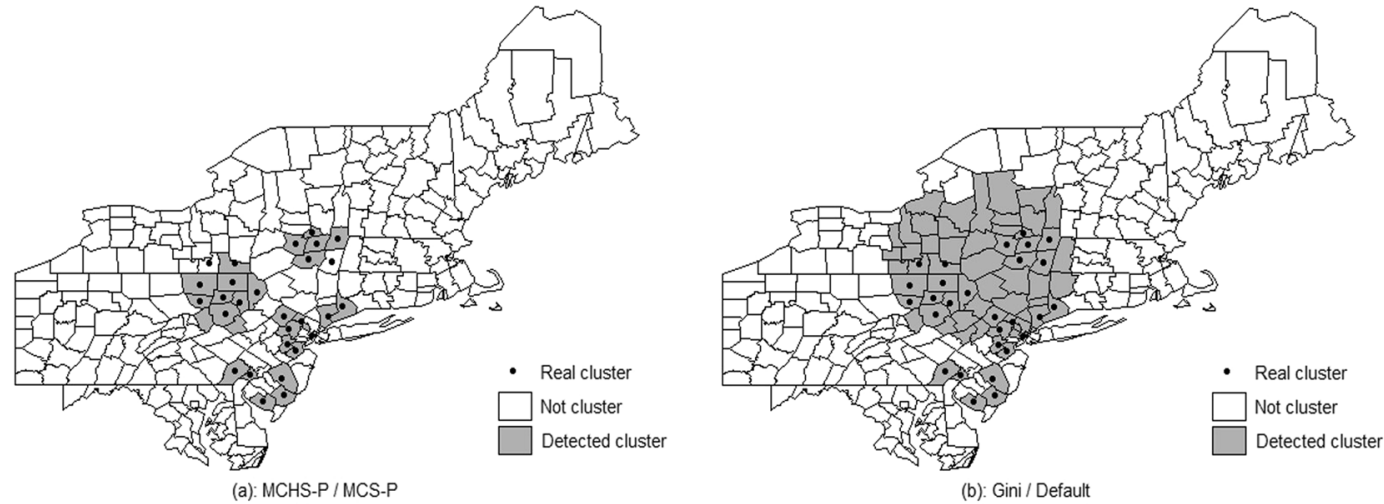

Figure 4. Comparison between the MCHS-P, the MCS-P, the default MWS and the Gini coefficient in one of simulated datasets in the additional scenario with a complex cluster model. Subfigure (a) is the detected result under the $2 \%$ MWS selected by both the MCHS-P and the MCS-P. Subfigure (b) is the detected result under the $50 \%$ MRWS selected by the Gini coefficient and the default 50\% MWS.

\begin{tabular}{|l|l|l|l|l|l|l|l|l|}
\hline Scenario type & \multicolumn{4}{|l}{ In 20 multi-cluster scenarios } & \multicolumn{3}{|l|}{ In 30 single-cluster scenarios } \\
\hline Measures & MCHS-P & MCS-P & Default & Gini & MCHS-P & MCS-P & Default & Gini \\
\hline Sensitivity & 19 & 0 & 1 & 0 & 26 & 28 & 4 & 4 \\
\hline Specificity & 0 & 20 & 0 & 0 & 26 & 30 & 26 & 26 \\
\hline PPV & 0 & 20 & 0 & 0 & 2 & 5 & 25 & 26 \\
\hline YDI & 20 & 0 & 1 & 1 & 22 & 28 & 2 & 2 \\
\hline Misclassification & 20 & 0 & 4 & 6 & 26 & 30 & 22 & 22 \\
\hline
\end{tabular}

Table 2. Frequency of the MCHS-P, MCS-P, 50\% MWS, and Gini coefficient obtaining the optimal result* in 20 multi-clusters scenarios and in 30 single-cluster scenarios for the five classic performance measures. *The optimal result is defined as the range that varies within 0.001 around the real optimal value of classic performance measure, such as the maximal sensitivity, specificity, PPV, YDI and the minimal misclassification. Therefore, in a given scenario, it is possible for more than one method to obtain the optimal result.

\begin{tabular}{|l|l|l|l|l|}
\hline Measures & MCHS-P & MCS-P & Default & Gini \\
\hline Sensitivity & 0.8207 & 0.8173 & 0.7365 & 0.8145 \\
\hline Specificity & 0.9944 & 0.9955 & 0.8989 & 0.9637 \\
\hline PPV & 0.9611 & 0.9672 & 0.6059 & 0.8281 \\
\hline YDI & 0.8151 & 0.8128 & 0.6354 & 0.7782 \\
\hline Misclassification & 0.0287 & 0.0282 & 0.1226 & 0.0561 \\
\hline
\end{tabular}

Table 3. Average classic performance measures over replicas from the MCHS-P, MCS-P, 50\% MWS and Gini coefficient in the complex scenario.

overall average values of the sensitivity, specificity, PPV, YDI and misclassification under the parameter selected using the MCHS-P show only a very slightly inferior values compared to the MCS-P $(0.0002,0.0005,0.0068$, and 0.0007 smaller and 0.0005 larger, respectively). Although the disadvantage of the MCHS-P relative to the MCS-P increases as the total case number and cluster size decrease, the largest disadvantages in the sensitivity, specificity, PPV, YDI and misclassification found in 600-rural- 1 are still very small $(0.0000,-0.0024,-0.0524,-0.0024$, and 0.0024 , respectively). Compared with the $50 \%$ MWS and the Gini coefficient, although the performance of the MCHS-P is poorer in several scenarios with a single high-RR and low-population cluster, the maximal difference is still very small, for example, 0.0007 , for both YDI and misclassification found in 600-rural-1. In addition, as shown in Table 2, both the MCHS-P and the MCS-P obtain the optimal result for both YDI and misclassification in more than 20 out of 30 scenarios, while the Gini coefficient obtains the optimal value for YDI in only 2 scenarios.

In the complex scenario, the $50 \%$ MWS performs poorly for detecting an overly large cluster including several small real clusters, in agreement with the previous study $6,17,30$. As shown in Table 3, the Gini coefficient improves the performance, reaching the values of $0.0780,0.0647,0.2222,0.1428$, and 0.0665 for sensitivity, specificity, PPV, YDI, and misclassification, respectively. Compared with the MCS-P and the MCHS-P, which have similar performance for the homogeneity between the clusters, the improvement due to the use of the Gini coefficient is still insufficient, e.g., on average, the MCHS-P and the MCS-P reduce 76.7\% misclassification relative to the default 


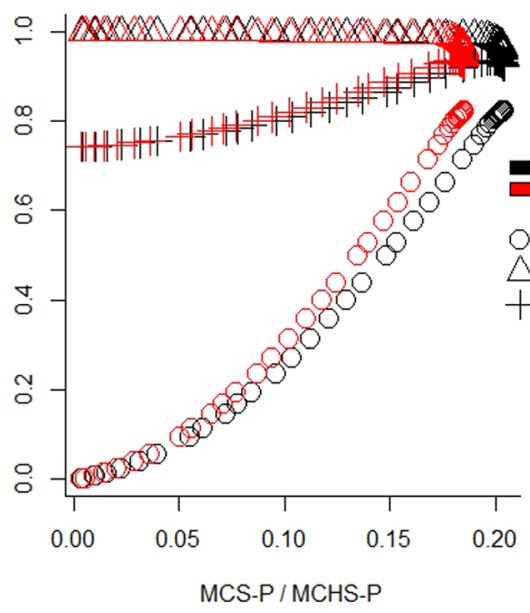

(a)

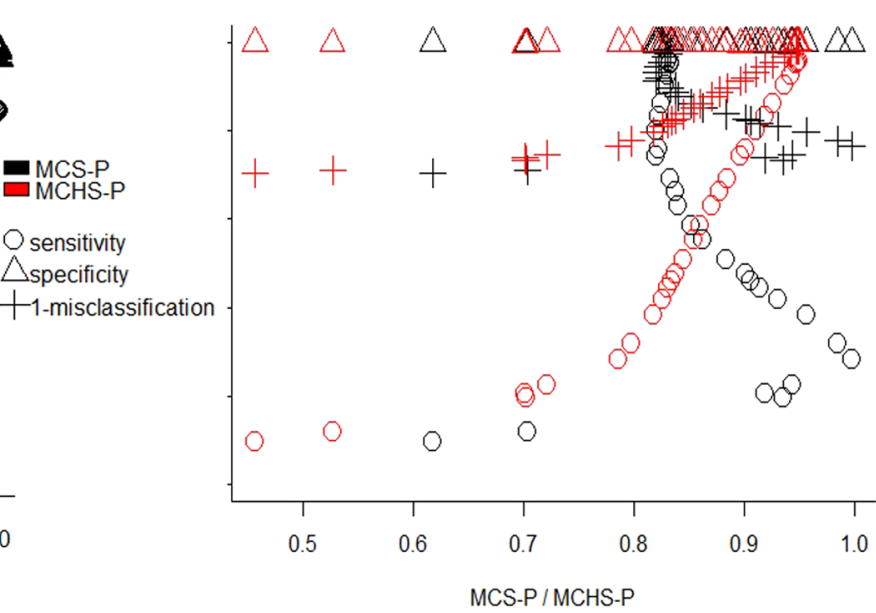

(b)

Figure 5. Relationship of the MCHS-P and the MCS-P with classical performance measures. Subfigure (a) is for a homogeneous scenario such as 6000-urban-16, and shows that both the MCHS-P and the MCS-P have a positive relationship with the classic performance measure. Subfigure $(\mathbf{b})$ is for a heterogeneous scenario such as 6000-three-16RR3.0-2.0-1.2, and it shows that the MCHS-P has a positive relationship with the classic performance measures, whereas the MCS-P has a nearly negative relationship with these performance measures.

MWS, while the reduction due to the Gini coefficient is $54.2 \%$; additionally, the performance of the Gini coefficient method is much more unstable than those of the MCHS-P and the MCS-P, especially for misclassification, PPV and specificity, as shown in Fig. 3. A further examination of the simulated datasets shows that the Gini coefficient selected 50\% MRWS as optimal in some cases and leads to the same detected results as the default MWS, giving high false positive rates. Figure 4 shows one such dataset out of the 10,000 simulated datasets in which the detected large cluster does indeed include the non-cluster region between the several small real clusters, further supporting the discussion of the Gini coefficient in the methodology section above.

The relationship of the MCHS-P and the MCS- $P$ with classic performance measures. In addition to the comparison of optimal MWS selected using the MCHS-P, MCS-P, Gini coefficient and default MWS, the relationships between the MCHS-P, MCS-P and classic performance measures such as sensitivity, specificity, PPV, YDI and misclassification, were evaluated to validate whether the selection of MWS using the MCHS-P can generally lead to detected results with better spatial accuracy, seen in Supplementary File 3.

Generally, as found previously, a larger MCS-P is related to better classic performance measures in scenarios with homogeneous and weakly heterogeneous clusters but is related to poorer performance in scenarios with heterogeneous clusters. By contrast, the MCHS-P is consistently positively related to the performance measures in all scenarios. Consistently high specificity and PPV were found in all scenarios similar to previous work ${ }^{17}$.

Several typical scenarios were selected for a detailed demonstration of the relationship of the MCHS-P and the MCS-P with classic performance measures and the difference between the MCHS-P and the MCS-P. In scenarios with homogeneous clusters, 6000-urban-16 was selected for a clear demonstration because its large population in clusters make the detected result sensitive to the MWS. Because PPV and YDI are almost equal to specificity and sensitivity in this scenario, respectively, only specificity, sensitivity and accuracy (1-misclassification) were presented. As shown in Fig. 5(a), both the MCHS-P and the MCS-P show positive relationships with sensitivity and accuracy, meaning that both the MCHS-P and the MCS-P will select the MWS with the optimal result when the clusters are homogeneous.

In scenarios with heterogeneous clusters, 600-two-1 was selected for its high heterogeneity between the clusters. Because the small population in the clusters makes it difficult to clearly present the relationship using a graphic plot, detailed values of the MCS-P, MCHS-P and performance measures were provided, shown in Supplementary File 2. the MWS with the largest the MCS-P has the lowest sensitivity, YDI and highest misclassification, whereas the MWS with the largest MCHS-P has the highest sensitivity, YDI and lowest misclassification. The specificity and PPV always stay at a rather high level and show little variation. On the other hand, because the benchmark datasets with large population in clusters have weak heterogeneity, for example 6000-three-3 and 600-three-3 shown in Table 1, we built an additional simulation scenario, available from Supplementary File 4, including clusters with relatively high heterogeneity and larger population to present the detailed relationship more clearly using a graphic plot. In this scenario, the cluster model has the same artificial clustering regions, the population at risk and total case number as those of 6000-three-16. Differently, the heterogeneity among the clusters was increased by using RRs of 3.0, 2.0 and 1.2 for the clusters located in rural, mixed and urban areas. The differences in RR between the clusters are much smaller than those in scenarios with simulated heterogeneous clusters, for example 190.16 in 600-two-1 and 2.37 in 600-two-16. This new scenario is abbreviated as 6000 -three16RR3.0-2.0-1.2. Similar to 6000-urban-16, PPV and YDI are also almost equal to the specificity and sensitivity, 
respectively. As shown in Fig. 5(b), the relationship of the MCS-P and the MCHS-P with classical performance measures shows that in scenarios with high heterogeneity, the MCHS-P showed a positive association with sensitivity and accuracy, whereas the MCS-P shows a negative association with these measures. This means that in the datasets with highly heterogeneous clusters, the MCHS-P still can select the optimal MWS while the MCS-P fails. Therefore, given the stable positive association to classic performance measures in both scenarios with homogeneous and heterogeneous clusters, a detected result with higher MCHS-P will also have better classic performance measures suggesting better spatial accuracy.

Practical study: comparison between the four methods. To provide examples of application of the MCHS-P, we employed two real datasets to compare the difference between the detected clusters obtained using the default parameters, selected by the MCHS-P, MCS-P, and Gini coefficient. The first data set is for female breast cancer which is a chronic disease and the second dataset is for measles which is an infectious disease that was used in a previous study ${ }^{17}$.

Female breast cancer mortality in the northeastern USA. The recent breast cancer dataset that is the same as those in the simulation study was obtained from the official website of the National Centre for Health Statistics and includes a total of 44,182 female deaths from breast cancer in 245 counties in the northeastern USA in $2011-2015^{31}$. The annual average total female population is $32,587,167$. Unlike the artificial clusters in simulated datasets, the real dataset shows more complex spatial pattern that reflects the characteristics of the breast cancer which is a chronic disease.

For the more complex characteristics in this practical dataset, 500 MWSs in the $0.1-50 \%$ range with an interval of $0.1 \%$ population at risk were set as the candidate parameters in order to obtain a more accurate result. Other settings are the same as those in the simulation study.

The 3.4\% and 5.5\% MWSs were selected using the MCS-P and the MCHS-P, respectively and the 30\% MRWS was selected using the Gini coefficient. The detected results obtained using the MCHS-P, MCS-P, default MWS (50\%) and Gini coefficient are shown in Fig. 6. The four detected results mainly differ in the southwestern region. With the default 50\% MWS, the SSS detected a large cluster covering over $45 \%$ of the total female population. Large number of low-mortality counties were included and led to an average RR of 1.1. The Gini coefficient shows better performance but still detected large regions as clusters in which the proportion of the female population reach $38 \%$ and the average $\mathrm{RR}$ is 1.12 . With limited public health resources, such a large detected cluster with flat RR may not provide accurate information for further interventions. The MCHS-P and the MCS-P tend to detect the clusters with smaller female populations, such as $22.6 \%$ and $20.0 \%$, and higher RR, such as 1.20 and 1.22 , respectively.

Comparing the results of the MCS-P and the MCHS-P, a total of 50 and 58 counties were detected as clusters using the MWSs of $3.4 \%\left(Z_{3.4}\right)$ and $5.5 \%\left(Z_{5.5}\right)$, respectively. Of these counties, 44 are identical. Table 4 shows that six counties were detected using $Z_{3.4}$ but were not detected using $Z_{5.5}$, and three of these had $R R$ values smaller than 1 . Fourteen counties were detected using $Z_{5.5}$ but were not detected using $Z_{3.4}$, and only two of them had RR values smaller than 1 . Figure 6 shows that the heterogeneity among the detected clusters is not high and the different counties detected as clusters using $Z_{3.4}$ and $Z_{5.5}$ are distributed around the identical counties. The eastern four counties labelled as B1 with relatively high mortality were not detected as clusters by $Z_{3.5}$, even though all of their eastern neighbouring counties were detected as clusters, suggesting that possible clusters were omitted using $\mathrm{Z}_{3.5}$. By contrast, they were detected by $\mathrm{Z}_{5.5}$. The counties labelled $\mathrm{B} 2$ and B3 display a similar performance to that of $\mathrm{B} 1$. The regions labelled $\mathrm{A} 1$ and $\mathrm{A} 4$ with low mortality were detected as clusters by $\mathrm{Z}_{3.4}$, suggesting incorrectly reported low-mortality regions. By contrast, they were excluded by $Z_{5.5}$. Although A2 and A3 with a relatively high mortality were omitted by $\mathrm{Z}_{5.5}, \mathrm{~A} 2$ and $\mathrm{A} 3$ include only two counties, and the heterogeneity among the clusters is low.

Measles incidence in henan province, china. The measles incidence data in Henan Province, China in May 2009 that was used to validate the MCS-P in selecting the $\mathrm{MWS}^{17}$ was obtained from the disease reporting system of China CDC. A total of 1,371 measles cases were reported in a population of $91,669,661$. The four methods, i.e., the MCHS-P, MCS-P, default MWS and Gini coefficient, had the same parameter set as that in the female breast cancer mortality dataset discussed above. As shown in Fig. 7, the default MWS (50\%) and the Gini coefficient obtained the same result and both detected a large region as clusters in which the proportion of the population to the total population reaches $34.7 \%$ but a low RR such as 1.96 . the MCHS-P and the MCS-P selected the same MWS (1.6\%) due to the weak heterogeneity between the clusters. They detected several small clusters with a $12.0 \%$ proportion of population and a high RR of 3.26 , obviously decreasing the false positive rate by excluding the counties with lower RR.

\section{Discussion}

Both the practical study and simulation study suggested that the detected result of the SSS is highly sensitive to the MWS. The commonly used default 50\% MWS reports relatively accurate clusters in dataset with clusters far away from each other, but reports an overly large cluster in datasets with clusters that are not far away, leading to a high false positive rate so that it can hardly provide accurate results. Although without any prior information, the Gini coefficient can improve the detected result by selecting a MRWS, but it is still likely to report a cluster that is larger than the real cluster. In particular, in datasets with complex cluster distributions, the Gini coefficient even has the same poor result as the default MWS. Although the MCS-P can select an appropriate MWS to avoid reporting such large clusters and improves the accuracy of the results in practical datasets, heterogeneous clusters in the applied spatial datasets may lead to unsatisfactory performance. In such cases, the modified MCHS-P 

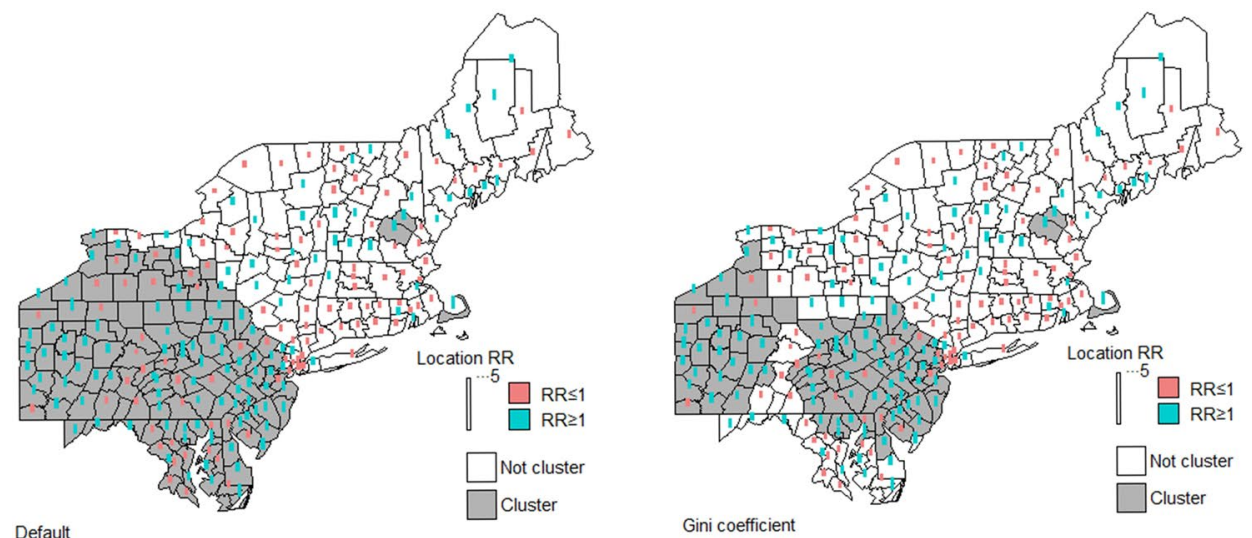

Default

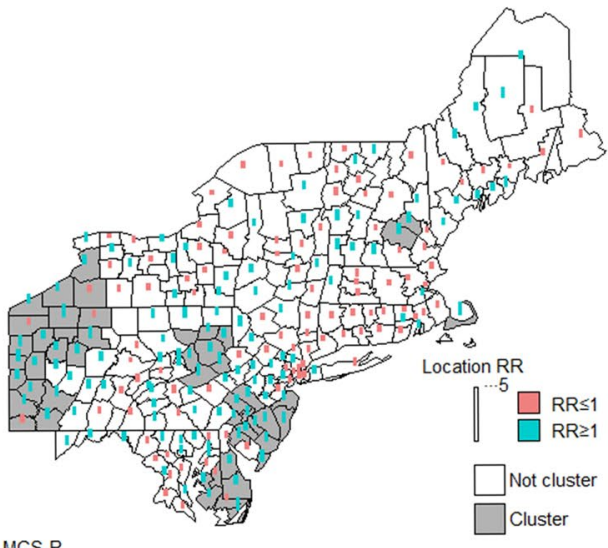

MCS-P
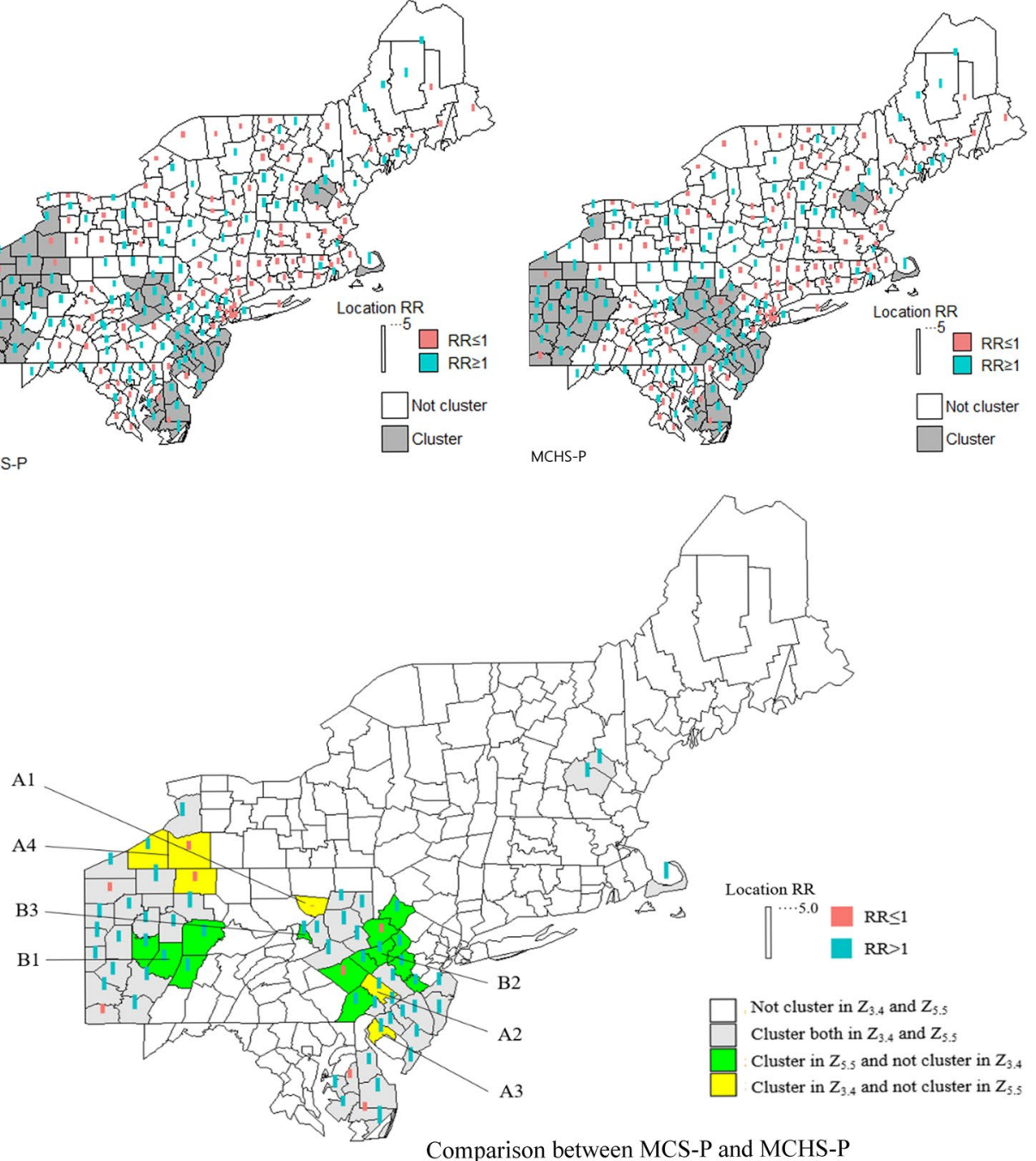

Figure 6. Detected clusters obtained using different parameters. The top four subfigures are for the default MWS (50\%) and 30\% MRWS selected by the Gini coefficient, the 3.4\% MWS selected by the MCS-P and the $5.5 \%$ MWS selected by the MCHS-P. The bottom subfigure shows the comparison between the results from the MCHS-P and the MCS-P.

showed better performance than the MCS-P in both simulation and practical datasets with heterogeneous clusters. Meanwhile, it showed no significant difference from the MCS-P by assuming a similar probability of event occurrence in contiguous clusters in order to limit overfitting. As the heterogeneity between clusters increases, the advantage of the MCHS-P over the MCS-P becomes even greater.

Similar to previous studies, in the scenarios with clusters very far away, specificity and PPV stay at high level ${ }^{6,17,27,32}$, so that the sensitivity and overall performance measures such as YDI and misclassification are of 


\begin{tabular}{|c|c|c|c|c|}
\hline $\begin{array}{l}\text { Found only in the } \\
\text { MCS-P/MCHS-P }\end{array}$ & Region & Code & Pop & RR \\
\hline \multirow{6}{*}{ MCS-P } & A1 & 42113 & 3038 & 0.01 \\
\hline & A2 & 42091 & 41794 & 1.116985 \\
\hline & A3 & 34033 & 33325 & 1.306212 \\
\hline & \multirow{3}{*}{ A4 } & 36009 & 39828 & \begin{tabular}{|l|}
0.888781 \\
\end{tabular} \\
\hline & & 36013 & 66959 & 1.10174 \\
\hline & & 42083 & 20874 & 0.953987 \\
\hline \multirow{14}{*}{ MCHS-P } & \multirow{4}{*}{ B1 } & 42005 & 34275 & 1.248418 \\
\hline & & \begin{tabular}{|l|}
42021 \\
\end{tabular} & 70657 & 1.284748 \\
\hline & & 42033 & 38982 & 1.21123 \\
\hline & & 42063 & 43982 & 1.09015 \\
\hline & \multirow{9}{*}{ B2 } & 34019 & 63792 & 1.005898 \\
\hline & & 34021 & 189129 & 1.002259 \\
\hline & & 34041 & 55027 & 1.139572 \\
\hline & & \begin{tabular}{|l|}
42011 \\
\end{tabular} & 210473 & \begin{tabular}{|l|}
0.988145 \\
\end{tabular} \\
\hline & & 42029 & 258927 & 1.074539 \\
\hline & & 42077 & 183185 & 1.144406 \\
\hline & & 42089 & 84994 & 0.910956 \\
\hline & & 42095 & 152641 & 1.063357 \\
\hline & & \begin{tabular}{|l|}
42103 \\
\end{tabular} & 28268 & 1.148192 \\
\hline & B3 & 42093 & 9582 & 1.30857 \\
\hline
\end{tabular}

Table 4. Different counties detected using 3.4\% and 5.5\% MWSs selected using the MCS-P and the MCHS-P. Note: RR values less than 1 are highlighted in bold.
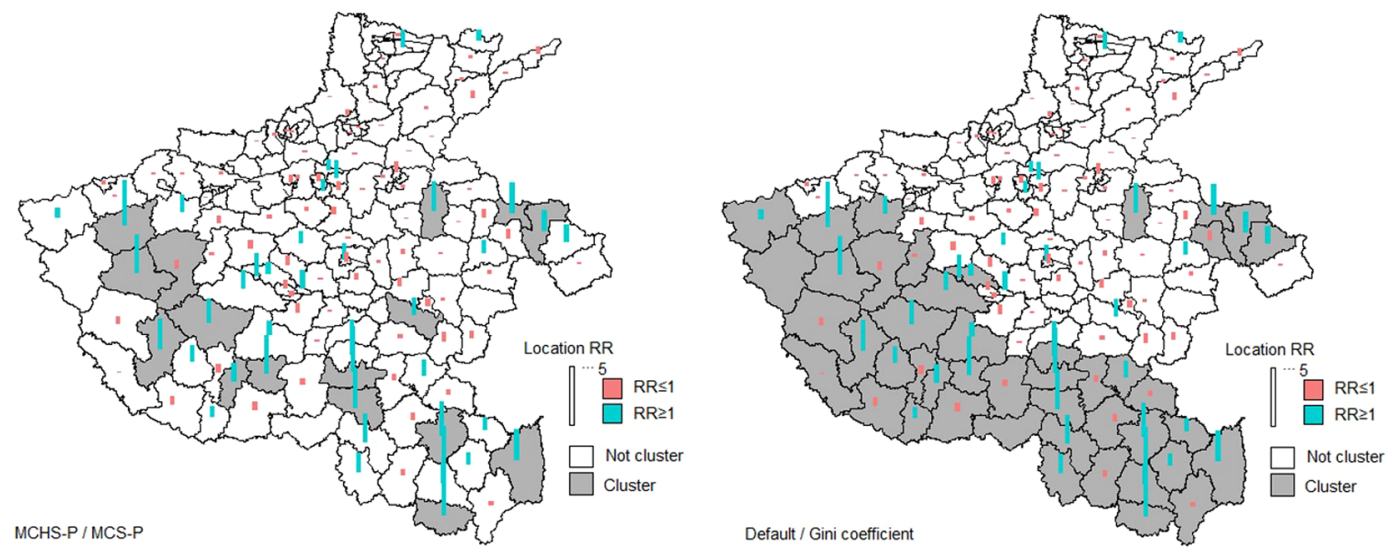

Figure 7. Detected clusters obtained using different parameters. The left subfigure is for the results obtained using 1.6\% MWS selected by the MCHS-P and the MCS-P, and the right subfigure is for the results obtained using the default MWS (50\%) and the MRWS (20-50\%) selected by the Gini coefficient.

more interest in most studies. In all simulation scenarios with heterogeneous clusters, the values of sensitivity, YDI and misclassification under the parameter selected using the MCHS-P were greatly improved compared to those obtained using the MCS-P. Moreover, higher heterogeneity leads to greater improvement of up to $540.27 \%$, $537.92 \%$ and $69.40 \%$, for sensitivity, YDI and misclassification, respectively. The correlation of the MCHS-P and the MCS-P with classical performance measures (Fig. 5) shows that in the datasets with little heterogeneity among the clusters, the results detected with a higher MCS-P and MCHS-P have better sensitivity, accuracy and YDI. Additionally, as the heterogeneity increases, the MCHS-P is still positively related to sensitivity, accuracy and YDI, whereas the relationship of the MCS-P with them becomes negative. Therefore, an appropriate parameter can be selected using the MCHS-P in spatial datasets with highly heterogeneous clusters for which the MCS-P will fail. In simulation datasets with homogeneous clusters, the performance of the MCHS-P is disadvantageous in some cases compared to the MCS-P because the MCHS-P may mistakenly identify high-risk regions caused by random fluctuation as a cluster for overfitting. However, the average disadvantage values for sensitivity, specificity, PPV, YDI, and misclassification are only $0.0002,0.0005,0.0068,0.0007$ and 0.0005 , respectively. Although the disadvantage increases with the decrease in the total case and cluster size because false clusters can be more easily detected in datasets with small cluster sizes and total case number, the largest disadvantage remains very slight $(0.0000,-0.0024,-0.0524,-0.0024$, and 0.0024 , respectively). These results suggest that assuming the similarity 
of contiguous clusters effectively limited overfitting in the MCHS-P and obtained a similar result with the MCS-P in spatial datasets with fairly homogeneous clusters.

Compared to the commonly selected default 50\% MWS and the Gini coefficient, the MCHS-P shows better performance in most scenarios. As the population in clusters and the number of clusters increase, meaning that the random fluctuation decreases, the advantage of the MCHS-P over the Gini coefficient and the default parameter increases, and especially in the additional simulation scenario with the complex spatial pattern, the average advantage values over the default MWS were $11.4 \%, 10.6 \%, 58.6 \%, 28.3 \%, 76.7 \%$ for sensitivity, specificity, PPV, YDI and misclassification, respectively, with the corresponding values of $0.7 \%, 3.1 \%, 16.1 \%, 4.7 \%$, and $48.9 \%$ over the Gini coefficient. In addition, the improvement due to the use of the Gini coefficient was found to be unstable, especially for misclassification, PPV and specificity in complex datasets. Only in the scenarios with high-RR and low-population single cluster does the MCHS-P have a slightly worse performance, for instance with the maximal difference for both misclassification and YDI of only 0.0007 observed in rural-600-1 as presented in Supplementary File 2.

In the case study of female breast cancer mortality data, most counties in the clusters detected using MWSs selected by the MCS-P and the MCHS-P are identical. Although the heterogeneity among the clusters is not high, the SSS's performance under the MWS selected using the MHCS-P is still better than that of the MCS-P. As the case result shows, the MCHS-P selected a larger MWS than the MCS-P did because the MCHS-P assumes that nonadjacent clusters are independent and each cluster can incorporate more neighbour counties for which the mortalities are closer to this cluster and are far away from other clusters. Therefore, more counties with a relatively high RR that are close to the clusters detected using the MCS-P are detected using the MCHS-P. In the two practical datasets, the Gini coefficient and the default MWS both reported clusters including many low-risk spatial units, which may provide poor information in fields with limited resources such as public health. Although the Gini coefficient led to some improvements in the first practical dataset, it made no improvements in the second practical dataset. Such unstable improvements of the Gini coefficient were also found in the complex scenario.

Besides, as we stated in the method section, since the denominator of MCS-P and MCHS-P is a constant regardless of the selected MWS, it is not necessary to calculate the denominator of MCS-P and MCHS-P when we use them to select the MWS in a practical dataset.

\section{Conclusion}

Most practical datasets may contain multiple clusters that are not so far away from each other, and the SSS with default MWS is much more likely to detect overly large clusters with poor accuracy. In this case, selecting an appropriate MWS is critical for the accurate performance of the SSS. Although the Gini coefficient and the MCS-P can be used to select a more appropriate MRWS or MWS to improve the detected result, the Gini coefficient may be unstable and obtain insignificant improvements. The MCS-P will select an inappropriate MWS in the dataset with highly heterogeneous clusters. By contrast, the improved MCHS-P will have stable and good performance. Additionally, as found for the relationship between the MCHS-P and the classic performance measures, results detected with a higher MCHS-P will show good performance for sensitivity, specificity, PPV, YDI and misclassification regardless of the homogeneity and heterogeneity between the clusters. Therefore, in the case without prior regarding true clusters or with the prior of heterogeneous clusters, the MCHS-P should be recommended for selecting an appropriate MWS for the SSS to obtain better accuracy. In the case with the prior that only homogeneous clusters exist, MCS-P should be employed to achieve best performance which has slight advantages over that using MCHS-P.

\section{Data availability}

- The simulation datasets used during this study are available on the website of SaTScan for the benchmark datasets: https://www.satscan.org/datasets/nebenchmark/index.html and the Supplementary Files 1 and 4 for the complex scenario and 6000-three-16RR3-2-1.2, respectively.

- The breast cancer dataset in the case study is available on the official website of the National Centre for Health Statistics: https://www.cdc.gov/cancer/uscs/dataviz/download_data.htm. And the measles incidence dataset is available from the corresponding author on reasonable request.

Received: 5 August 2019; Accepted: 4 March 2020;

Published online: 17 March 2020

\section{References}

1. Kulldorff, M. A spatial scan statistic. Communications in Statistics-Theory and Methods 26, 1481-1496, https://doi. org/10.1080/03610929708831995 (1997).

2. K. M, Selected Applications by Field of Study. https://www.satscan.org/references.html\#Selected\%20Applications\%20by\%20 Field\%20of\%20Study (2019).

3. Wan, N., Zhan, F. B., Lu, Y. \& Tiefenbacher, J. P. Access to healthcare and disparities in colorectal cancer survival in Texas. Health \& Place 18, 321-329, https://doi.org/10.1016/j.healthplace.2011.10.007 (2012).

4. Cuadros, D. F. \& Abu-Raddad, L. J. Spatial variability in HIV prevalence declines in several countries in sub-Saharan. Africa. Health \& Place 28, 45-49, https://doi.org/10.1016/j.healthplace.2014.03.007 (2014).

5. Gao, F., Foster, M. \& Liu, Y. Disability concentration and access to rehabilitation services: a pilot spatial assessment applying geographic information system analysis. Disability and Rehabilitation 41, 2468-2476, https://doi.org/10.1080/09638288.2018.1468931 (2019).

6. Rodrigues Ribeiro, S. H. \& Costa, M. A. Optimal selection of the spatial scan parameters for cluster detection: A simulation study. Spatial and Spatio-Temporal Epidemiology 3, 107-120, https://doi.org/10.1016/j.sste.2012.04.004 (2012).

7. Tango, T. \& Takahashi, K. A flexibly shaped spatial scan statistic for detecting clusters. International journal of health geographics 4 , 11-11, https://doi.org/10.1186/1476-072x-4-11 (2005). 
8. Chen, J., Roth, R. E., Naito, A. T., Lengerich, E. J. \& Maceachren, A. M. Geovisual analytics to enhance spatial scan statistic interpretation: an analysis of U.S. cervical cancer mortality. International journal of health geographics 7, 57-57, https://doi. org/10.1186/1476-072x-7-57 (2008).

9. Yiannakoulias, N., Rosychuk, R. J. \& Hodgson, J. Adaptations for finding irregularly shaped disease clusters. International Journal of Health Geographics 6, https://doi.org/10.1186/1476-072x-6-28 (2007).

10. Coulston, J. W. \& Riitters, K. H. Geographic analysis of forest health indicators using spatial scan statistics. Environmental Management 31, 764-773, https://doi.org/10.1007/s00267-002-0023-9 (2003).

11. Weisent, J., Rohrbach, B., Dunn, J. R. \& Odoi, A. Detection of high risk campylobacteriosis clusters at three geographic levels. Geospatial Health 6, 65-76, https://doi.org/10.4081/gh.2011.158 (2011).

12. Marek, L., Tucek, P. \& Paszto, V. Using geovisual analytics in Google Earth to understand disease distribution: a case study of campylobacteriosis in the Czech Republic (2008-2012). International Journal of Health Geographics 14 https://doi.org/10.1186/1476072x-14-7 (2015).

13. Forand, S. P., Talbot, T. O., Druschel, C. \& Cross, P. K. Data quality and the spatial analysis of disease rates: congenital malformations in New York State. Health \& Place 8, 191-199, https://doi.org/10.1016/s1353-8292(01)00037-5 (2002).

14. Costa MA, K. M. Scan statistics: methods and applications. Birkkäuser: Statistics for Industry and Technology; 2009. p. 129-52[chapter 6]. (2009)

15. Chaput, E. K., Meek, J. I. \& Heimer, R. Spatial analysis of human granulocytic ehrlichiosis near Lyme, Connecticut. Emerging Infectious Diseases 8, 943-948, https://doi.org/10.3201/eid0809.020103 (2002).

16. Amin, R., Nelson, A. \& McDougall, S. A Spatial Study of the Location of Superfund Sites and Associated Cancer Risk. Statistics and Public Policy 5, 1-9, https://doi.org/10.1080/2330443x.2017.1408439 (2017).

17. Ma, Y., Yin, F., Zhang, T., Zhou, X. A. \& Li, X. Selection of the Maximum Spatial Cluster Size of the Spatial Scan Statistic by Using the Maximum Clustering Set-Proportion Statistic. Plos One 11, https://doi.org/10.1371/journal.pone.0147918 (2016).

18. Han, J. et al. Using Gini coefficient to determining optimal cluster reporting sizes for spatial scan statistics. International Journal of Health Geographics 15, 27 (2016).

19. Kim, S. \& Jung, I. Optimizing the maximum reported cluster size in the spatial scan statistic for ordinal data. Plos One 12, e0182234 (2017).

20. Tobler, W. R. Computer movie simulating urban growth in detroit region. Economic Geography 46, 234-240, https://doi. org/10.2307/143141 (1970).

21. Kulldorff, M., Tango, T. \& Park, P. J. Power comparisons for disease clustering tests. Computational Statistics \& Data Analysis 42, 665-684, https://doi.org/10.1016/s0167-9473(02)00160-3 (2003).

22. Costa, M. A., Assuncao, R. M. \& Kulldorff, M. Constrained spanning tree algorithms for irregularly-shaped spatial clustering. Computational Statistics \& Data Analysis 56, 1771-1783, https://doi.org/10.1016/j.csda.2011.11.001 (2012).

23. Kulldorff, M., Huang, L., Pickle, L. \& Duczmal, L. An elliptic spatial scan statistic. Statistics in Medicine 25, 3929-3943, https://doi. org $/ 10.1002 / \operatorname{sim} .2490$ (2006).

24. Duczmal, L., Kulldorff, M. \& Huang, L. Evaluation of spatial scan statistics for irregularly shaped clusters. Journal of Computational and Graphical Statistics 15, 428-442, https://doi.org/10.1198/106186006x112396 (2006)

25. K. M, Northeastern USA Benchmark Data, Purely Spatial 2018. https://www.satscan.org/datasets/nebenchmark/index.html (2019).

26. Huang, L., Pickle, L. W. \& Das, B. Evaluating spatial methods for investigating global clustering and cluster detection of cancer cases. Statistics in Medicine 27, 5111-5142, https://doi.org/10.1002/sim.3342 (2008)

27. Lima, M. S. D., Santos, V. S. D. \& Duczmal, L. H. A spatial scan statistic for beta regression. Spatial Statistics 18, 444-454, https://doi. org/10.1016/j.spasta.2016.09.003 (2016)

28. Wiegand, R. E. et al. A Persistent Hotspot of Schistosoma mansoni Infection in a Five-Year Randomized Trial of Praziquantel Preventative Chemotherapy Strategies. Journal of Infectious Diseases 216, 1425-1433, https://doi.org/10.1093/infdis/jix496 (2017).

29. Jung, I., Kulldorff, M. \& Richard, O. J. A spatial scan statistic for multinomial data. Statistics in Medicine 29, 1910-1918, https://doi. org/10.1002/sim.3951 (2010).

30. Chen, J., Roth, R. E., Naito, A. T., Lengerich, E. J. \& Maceachren, A. M. Geovisual analytics to enhance spatial scan statistic interpretation: an analysis of U.S. cervical cancer mortality. International Journal of Health Geographics 7, 1-18 (2008).

31. United States Cancer Statistics. USCS_1999_2015_ASCII.zip. https://www.cdc.gov/cancer/uscs/dataviz/download_data.htm (2018).

32. Moraga, P. \& Montes, F. Detection of spatial disease clusters with LISA functions. Statistics in Medicine 30, 1057-1071, https://doi. org/10.1002/sim.4160 (2011).

\section{Acknowledgements}

This study was supported by the National Natural Science Foundation of China (No. 81803332, No. 81602935 and No. 81872713), the Sichuan Science \& Technology Program (No. 2018 SZ0284 and No. 2019YFS0471), the Chengdu Science \& Technology Bureau (No. 2018-YF05-01265-SN) and the Postdoctoral Research foundation of Sichuan University (No. 2018SCU12012). All the funders had no role in the study design, data collection and analysis, the decision to publish, or preparation of the manuscript.

\section{Author contributions}

All authors contributed to the conception and design of this study. W.W., Y.M., X.X. and S.C. performed the study. W.W., T.Z. and Y.M. wrote the paper. Y.M., T.Z. and Y.F. obtained the funding.

\section{Competing interests}

The authors declare no competing interests.

\section{Additional information \\ Supplementary information is available for this paper at https://doi.org/10.1038/s41598-020-61829-y.}

Correspondence and requests for materials should be addressed to Y.M.

Reprints and permissions information is available at www.nature.com/reprints.

Publisher's note Springer Nature remains neutral with regard to jurisdictional claims in published maps and institutional affiliations. 
(c) (i) Open Access This article is licensed under a Creative Commons Attribution 4.0 International License, which permits use, sharing, adaptation, distribution and reproduction in any medium or format, as long as you give appropriate credit to the original author(s) and the source, provide a link to the Creative Commons license, and indicate if changes were made. The images or other third party material in this article are included in the article's Creative Commons license, unless indicated otherwise in a credit line to the material. If material is not included in the article's Creative Commons license and your intended use is not permitted by statutory regulation or exceeds the permitted use, you will need to obtain permission directly from the copyright holder. To view a copy of this license, visit http://creativecommons.org/licenses/by/4.0/.

(C) The Author(s) 2020 